\title{
Dependence of NAO variability on coupling with sea ice
}

\author{
Courtenay Strong • Gudrun Magnusdottir
}

Received: 21 September 2009/Accepted: 21 January 2010/Published online: 14 April 2010

(C) The Author(s) 2010. This article is published with open access at Springerlink.com

\begin{abstract}
The variance of the North Atlantic Oscillation index (denoted $N$ ) is shown to depend on its coupling with area-averaged sea ice concentration anomalies in and around the Barents Sea (index denoted $B$ ). The observed form of this coupling is a negative feedback whereby positive $N$ tends to produce negative $B$, which in turn forces negative $N$. The effects of this feedback in the system are examined by modifying the feedback in two modeling frameworks: a statistical vector autoregressive model $\left(F_{\mathrm{VAR}}\right)$ and an atmospheric global climate model $\left(F_{\mathrm{CAM}}\right)$ customized so that sea ice anomalies on the lower boundary are stochastic with adjustable sensitivity to the model's evolving $N$. Experiments show that the variance of $N$ decreases nearly linearly with the sensitivity of $B$ to $N$, where the sensitivity is a measure of the negative feedback strength. Given that the sea ice concentration field has anomalies, the variance of $N$ goes down as these anomalies become more sensitive to $N$. If the sea ice concentration anomalies are entirely absent, the variance of $N$ is even smaller than the experiment with the most sensitive anomalies. Quantifying how the variance of $N$ depends on the presence and sensitivity of sea ice anomalies to $N$ has implications for the simulation of $N$ in global climate models. In the physical system, projected changes in sea ice thickness or extent could alter the sensitivity of $B$ to $N$, impacting the within-season variability and hence predictability of $N$.
\end{abstract}

C. Strong $(\bowtie)$

University of Utah, Salt Lake City, Utah 84112, USA

e-mail: court.strong@utah.edu

G. Magnusdottir

University of California, Irvine, Irvine, CA 92697-3100, USA

\section{Introduction}

Physical reasoning suggests that winter sea ice variability over the North Atlantic should be sensitive to the overlying atmospheric circulation since the latter can generate anomalies of sea ice velocity, atmospheric heat transport, and oceanic heat transport. As an example of this coupling, the upward trend of the North Atlantic Oscillation (NAO) index $(N)$ from the 1960s through the mid-1990s increased the rate of winter sea ice retreat over the North Atlantic (Deser 2000; Venegas and Mysak 2000; Rigor et al. 2002; Hu et al. 2002; Liu and Curry 2004; Rothrock and Zhang 2005; Ukita et al. 2007). Since then, the NAO trend has reversed, and an overall downward trend in total sea ice extent is emerging that appears to be anthropogenic (Johannessen et al. 2004) and accelerating in summer (e.g. Comiso 2006; Serreze et al. 2007). There nonetheless remains, superimposed on this overall downward trend in sea ice extent, a measurable signature of forcing by atmospheric circulation variability (Comiso 2006; Maslanik et al. 2007; Francis and Hunter 2004; Deser and Teng 2008).

A substantial fraction of this atmospheric forcing is connected to the NAO, whose imprint is discernible as wind-driven sea ice extent anomalies in daily data (Kimura and Wakatsucchi 2001), and ice motion and thickness anomalies in multi-year satellite records (Kwok et al. 2005). During positive NAO, sea ice concentrations in the Barents Sea tend to be lower than average in association with increased temperatures related to enhanced atmospheric and oceanic heat transport (Yamamoto et al. 2006; Wang et al. 2000; Liu and Curry 2004). Koenigk et al. (2009) recently used a fully coupled model to show that that sea ice concentrations within the Barents Sea are most sensitive to wind-driven sea ice transport, with oceanic heat 
transport playing a small role in interannual variations and a large role in variations on longer time scales.

NAO-driven sea ice variations project strongly onto, and are likely largely responsible for, the leading pattern of North Atlantic sea ice concentration variability (Deser et al. 2000). This leading pattern consists of a dipole pattern of oppositely signed concentration anomalies in the Labrador and Barents Seas, where Barents Sea concentrations are lower during positive NAO. We refer to this variability pattern as the Greenland Sea-ice Dipole (GSD).

Modeling studies have shown that a positive GSD-like sea ice pattern sustained from December through April will generate a negative NAO-like hemispheric-scale response (Magnusdottir et al. 2004; Deser et al. 2004; Alexander et al. 2004; Kvamsto et al. 2004). These results evidence the presence of a negative feedback since the positive NAO produces a positive GSD pattern. Deser et al. (2007) showed that this negative feedback begins as a baroclinic response localized to the forcing that reaches peak intensity in 5-10 days and persists for 2-3 weeks. If the GSD pattern is sustained beyond several weeks, the atmosphere develops a larger-scale equivalent barotropic response resembling the negative polarity of the Northern Annular Mode, which is maintained primarily by nonlinear transient fluxes of eddy vorticity (Deser et al. 2007) related in part to changes in Rossby wave breaking (Strong and Magnusdottir 2010b).

There is evidence of non-stationarity in the association between sea ice and the NAO related to multi-decadal external forcing. Modeling studies show, for example, a strong impact of the North Atlantic meridional overturning circulation (MOC) on sea ice in the Arctic Ocean and Barents Sea (Delworth et al. 1997; Jungcalus et al. 2005). Reconstructing North Atlantic sea ice extent back to 1800 , Fauria et al. (2009) found weak running correlations prior to 1950 and significant correlations thereafter. For the period 1978-2007, Strong et al. (2009) (hereafter, SMS) detected significant negative feedback between winter sea ice and the NAO at weekly time scales using satellite observations of sea ice concentration, atmospheric reanalysis data, and the testable definitions of causality and feedback developed by Granger (1969). For interannual and longer time scales, Strong and Magnusdottir (2010a) examined multi-model ensemble simulations of the twentieth to twenty-third centuries, and found that an NAO-driven pattern of sea ice variabilty will persist but change somewhat in form as the ice edge retreats under projected global warming.

The present manuscript is focused on how the feedback between sea ice and the NAO affects the variance of sea ice and the variance of the NAO index. If, for example, we turn off the feedback or double the sensitivity of the feedback, how is the variance of the NAO index affected?
To quantify the effects of the sea ice-NAO feedback, we use two observationally-motivated models of the sea iceNAO system. The first model is based on the vectorautoregressive statistical model used in SMS. The second model is an atmospheric global climate model modified so that sea ice anomalies on the lower boundary are stochastic with adjustable sensitivity to the model's evolving NAO index. Our observational data are described in Sect. 2, followed by our modeling methods (Sect. 3), Results (Sect. 4), and a Summary and Discussion (Sect. 5).

\section{Data}

Consistent with SMS, we defined the NAO index $N$ based on the leading empirical orthogonal function (EOF) of weekly mean NCEP/NCAR reanalysis sea level pressure data for the 21-week extended winter from 4 December through 23 April for years 1978-2008. Data were detrended, deseasonalized, and restricted to the domain used in Hurrell $(1995)\left(20^{\circ}-80^{\circ} \mathrm{N}\right.$ and $\left.90^{\circ} \mathrm{W}-40^{\circ} \mathrm{E}\right)$. A portion of this EOF is contoured in Fig. 1.

SMS used a sea ice index $(G)$ based on the GSD pattern. Here, we simplify this by focusing on the Barents Sea center of action since it accounts for nearly the entire feedback signal detected by Magnusdottir et al. (2004). We define an index $B$ which is the area-weighted, weeklymean, sea ice concentration anomaly within the region outlined in Fig. 1, where the anomaly is relative to the long term mean for that week. This definition for $B$ yields an intuitive sign convention whereby high $B$ corresponds to anomalously high sea ice concentrations over the Barents Sea, but we note that the $\operatorname{sign}$ of $B$ is opposite to the sign of the GSD index, so the negative feedback in this system is positive $N \rightarrow$ negative $B \rightarrow$ negative $N$

as shown in Sect. 4.1.

The $B$ index is calculated using National Snow and Ice Data Center (NSIDC) sea ice concentrations derived from Nimbus-7 Scanning Multichannel Microwave Radiometer and Defense Meteorological Satellite Program Special Sensor Microwave/Imager radiances (Cavalieri et al. 2008). These sea ice data are on a $25-\mathrm{km}$ grid nominally once every 2 days for 1978-1986 and once daily for 1987 to present. We do not include winter 1987-1988 in our study because of a data gap.

\section{Modeling}

We use two modeling frameworks to explore how feedback between sea ice and the NAO affects the variance of sea ice and the variance of the NAO. The first framework is a 

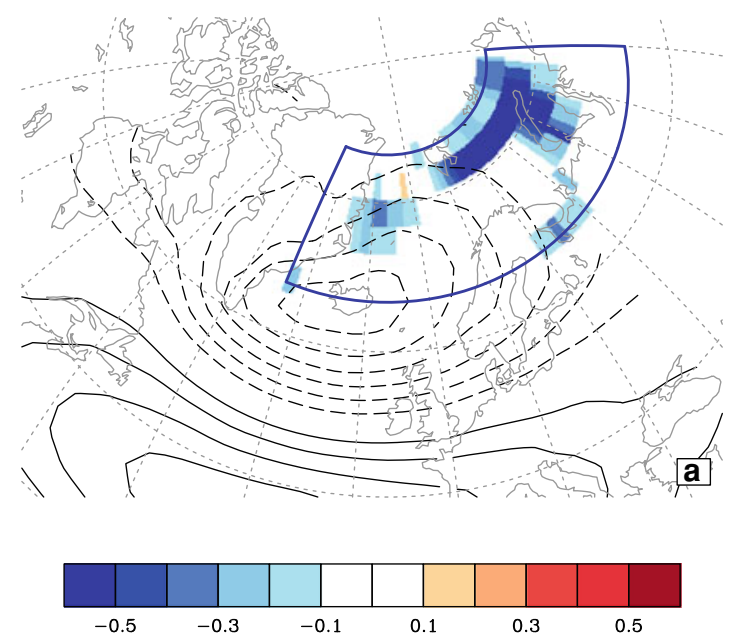

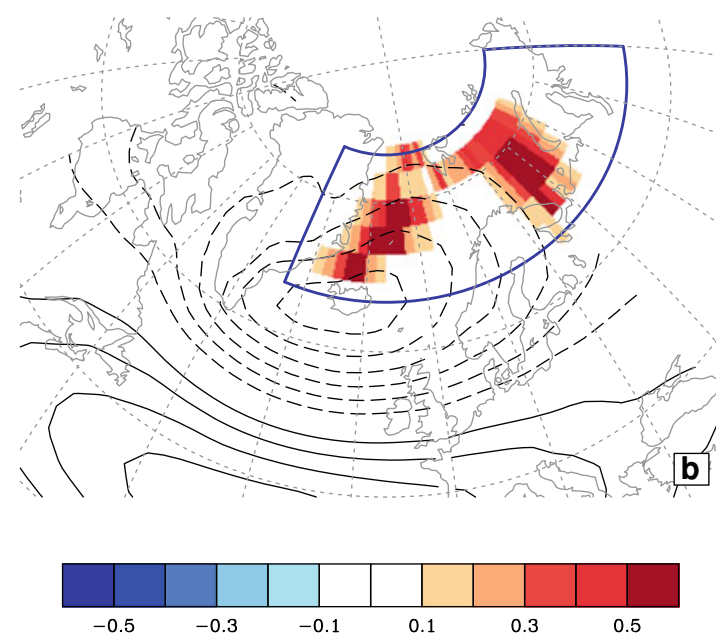

Fig. 1 Shading shows example sea ice anomaly fields from the "B-file" valid for (a) January with $-3.5 \leq B<-2.5$ and (b) February with $2.5 \leq B<3.5$. The EOF of the NAO is contoured with negative values dashed and the zero contour suppressed

linear statistical model coupling $N$ and $B$ as a vectorautoregressive (VAR) process (Sect. 3.1, framework denoted $\left.F_{\mathrm{VAR}}\right)$. The second framework couples a linear stochastic model of $B$ to the NCAR Community Atmosphere Model (CAM) Version 3.0 (Sect. 3.2, framework denoted $\left.F_{\mathrm{CAM}}\right)$. The simplicity of $F_{\mathrm{VAR}}$ allows us to run many long experiments with minimal computational expense, and to obtain explicit expressions for how the variances of $N$ and $B$ are affected by feedback. The $F_{\text {VAR }}$ results provide a view of system behavior based on a linear framework, and we compare these linear results to analogous experiments in the $F_{\mathrm{CAM}}$ system which is solving nonlinear partial differential equations to determine $N$.

\subsection{Statistical model}

In SMS, we studied feedback between sea ice and the NAO using a VAR model that included contemporaneous as well as lagged effects. For our purposes here, we maintain lag order $p$, simplify the model by excluding contemporaneous effects, and introduce "feedback scaling parameters" $g$ and $h$ to be used when experimenting with the model. Denoting $N$ during week $t$ by $N_{t}$, and $B$ during week $t$ by $B_{t}$, we write $F_{\text {VAR }}$ as

$$
\left(\begin{array}{c}
B_{t} \\
N_{t}
\end{array}\right)=\sum_{i=1}^{p}\left(\begin{array}{cc}
\alpha_{i} & g \beta_{i} \\
h \eta_{i} & \phi_{i}
\end{array}\right)\left(\begin{array}{c}
B_{t-i} \\
N_{t-i}
\end{array}\right)+\left(\begin{array}{c}
\varepsilon_{B t} \\
\varepsilon_{N t}
\end{array}\right)
$$

where $B_{t}$ and $N_{t}$ are stationary with zero mean, and $\varepsilon_{B t}$ and $\varepsilon_{N t}$ are uncorrelated white noise disturbances with respective standard deviations $\sigma_{B}$ and $\sigma_{N}$. The parameters $g$ and $h$ govern, respectively, how sensitive $B$ is to $N$ and how sensitive $N$ is to $B$. In general, and particularly when fitting $F_{\mathrm{VAR}}$ to observations, $g=h=1$. The feedback scaling parameters may be given values other than 1 for the purpose of experimentation aimed at determining the response of the system to a stronger (e.g., $g=1.5$ ) or weaker feedback (e.g., $g=0.5$ ). We can write (2) compactly as

$\mathbf{x}_{t}=\sum_{i} \phi_{i} \mathbf{x}_{t-i}+\boldsymbol{\varepsilon}_{t}$.

To determine the appropriate order $p$, we fit the model to observations at order $p$ (i.e., "unrestricted") and compare it to the model fit at order $p-1$ (i.e., "restricted"). Where we detect a significant degradation in model strength going from $p$ to $p-1$, we declare $p$ to be the appropirate model order. Significant degradations in model strength are tested for using the log-likelihood ratio given by Sims (1980)

$L \equiv(T-c)\left(\log \left|\boldsymbol{\Sigma}_{r}\right|-\log \left|\boldsymbol{\Sigma}_{u}\right|\right)$

where $T$ is the number of use-able observations, $c$ the maximum number of regressors in the longest equation, and $\left|\boldsymbol{\Sigma}_{u}\right|$ and $\left|\boldsymbol{\Sigma}_{r}\right|$ are the determinants of the covariance matrices of the unrestricted and restricted model's residuals, respectively.

To quantify the variance of $N$ and the variance of $B$ in $F_{\mathrm{VAR}}$, we want expressions for the $2 p+1$ covariance matrices

$\boldsymbol{\Gamma}(m) \equiv \mathrm{E}\left(\mathbf{x}_{t+m} \mathbf{x}_{t}^{\mathrm{T}}\right)=\left(\begin{array}{ll}\gamma_{B B}(m) & \gamma_{B N}(m) \\ \gamma_{N B}(m) & \gamma_{N N}(m)\end{array}\right)$
$m=0, \pm 1, \ldots, \pm p$

where $m$ is lag, $(\cdot)^{T}$ denots transpose, $\mathrm{E}(\cdot)$ denotes expectation, and $\gamma_{B N}(m)$ the covariance where $N$ leads $B$ by $m$ weeks. For convenience, we denote the variances of $N$ and $B$ as $\gamma_{N N}$ and $\gamma_{B B}$. The $2 p+1$ matrices in (5) have a total of $8 p+4$ elements, but at most $4 p+3$ of them are unique since $\boldsymbol{\Gamma}(-m)=\boldsymbol{\Gamma}^{T}(m)$ and $\gamma_{N B}(0)=\gamma_{B N}(0)$. To get $4 p+3$ linear equations for these $4 p+3$ unique matrix 
elements, we post-multiply (3) by $\mathbf{x}_{t-j}^{T}, j=0,1, \ldots, p$ and take expectations, yielding (e.g., Brockwell and Davis 1996)

$$
\begin{aligned}
& \boldsymbol{\Gamma}(i)=\sum_{j=1}^{p} \boldsymbol{\phi}_{j} \boldsymbol{\Gamma}(i-j), \quad i=1,2, \ldots, p \\
& \boldsymbol{\Gamma}(0)=\sum_{j=1}^{p} \boldsymbol{\phi}_{j} \boldsymbol{\Gamma}(-j)+\mathbf{\Upsilon}
\end{aligned}
$$

where $\mathbf{Y}$ is the covariance matrix of $\boldsymbol{\varepsilon}_{t}$. Numerically solving the linear system (6-7) yields all the unique elements of (5), and we will be focusing primarily on the variances $\gamma_{N N}$ and $\gamma_{B B}$. As we will show in Sect. 4.2, the statistical properties of simulations from $F_{\mathrm{VAR}}$ converge to the solutions to (6-7) for large sample size.

\subsection{Atmospheric global climate model}

As described above, $F_{\mathrm{VAR}}$ couples a linear expression for $N$ to a linear expression for $B$ where the latter is written

$B_{t}=\sum_{i=1}^{p}\left(\alpha_{i} B_{t-i}+g \beta_{i} N_{t-i}\right)+\varepsilon_{B t}$.

$F_{\text {CAM }}$ retains (8) as the linear expression for $B$, but couples it to CAM. To acheive this coupling, we wrote a parallelized module for CAM that introduces a weekly sea ice concentration anomaly according to Eq. 8, but with the values of weekly mean $N$ calculated from the sea level pressure fields being generated within CAM during the run. The module requires three input files: (1) the spatial pattern of the NAO obtained from a long unforced run analyzed the same way as the observational NAO (Sect. 2), (2) a weekly climatology of sea level pressure based on a long unforced run, and (3) a " $B$-file" containing mapped sea ice concentration anomalies for a range of $B$ values and months.

The sea ice concentration anomalies in the $B$-file are given on the model's latitude-longitude grid, and are specified as a function of month and the index $B$. Each anomaly map is a composite of NSIDC sea ice concentration anomaly observations grouped by the five months December through April, and seven $B$ index bins centered around the integers $-3,-2, \ldots, 3$. As illustrative examples, Fig. 1a shows the composite sea ice anomaly for all January observations with $B$ indices in the bin centered on $B=-2$, and Fig. $1 \mathrm{~b}$ shows the composite sea ice anomaly for all February observations with $B$ indices in the bin centered on $B=3$. Experimentation with $F_{\text {CAM }}$ using more idealized, smooth anomalies produced results similar to those presented here.

Week $t=1$ is defined as the first seven days of model integration, and $F_{\mathrm{CAM}}$ specifies the preceding $p$ weeks as initial conditions. When the model initializes, it therefore sets $t=1$ and does the following:

1. Set initial conditions $B_{t=1-i}=0$ for $i=1,2, \ldots, p$.

2. Set initial conditions $N_{t=1-i}=c$ for $i=1,2, \ldots, p$ where $c$ is the value of the NAO index calculated from the sea level pressure field in the initial condition file.

3. Calculate $B_{1}$ using the $2 p$ initial conditions and Eq. 8 .

4. Determine the sea ice concentration anomaly field to be applied during week $t=1$ by going to the current model month in the $B$-file and linearly interpolating the sea ice concentration anomaly at each grid point as a function of $B$ to the value $B_{1}$.

The module then takes the steps required to perform the following every seven days beginning on week $t=2$ :

1. Calculate $N_{t-1}$ using the preceding week's sea level pressure fields, which the model has stored, weighted by the square root of the grid area, deseasonalized, and projected onto the spatial pattern of the NAO.

2. Calculate $B_{t}$ from Eq. 8 using $B_{t-i}$ and $N_{t-i}$ for $i=1,2, \ldots, p$.

3. Determine the sea ice concentration anomaly field to be applied during next seven days by going to the current model month in the $B$-file and linearly interpolating the sea ice concentration anomaly at each grid point as a function of $B$ to the value $B_{t}$.

For the sea ice concentration anomalies used in our experiments, going to a specific month in the $B$-file produces anomaly patterns that are similar to those obtained by performing a more expensive bi-linear interpolation as a function of month and $B_{t}$.

$F_{\text {CAM }}$ can be thought of as a modeling framework that is intermediate between running CAM coupled to a full ice model and running CAM forced by sea ice linearly interpolated from a climatology. This intermediate framework is useful for our purposes because we can explicitly control aspects of $B$ including whether, and to what degree, $B$ is sensitive to variations in CAM's evolving $N$.

\subsection{Experiments}

We designed our experiments to uncover the effects of feedback in the $B$ and $N$ system. In the experiments, we varied the values of the feedback scaling parameters $g$ and $h$ as shown in Table 1. Our control case (CTL) corresponds conceptually to the observed system, with $N$ and $B$ having realistic sensitivity to one another (i.e., $g=h=1$ ). For the CTL case, $B$ evolved as a vector autoregressive process sensitive to the past states of itself, the past states of $N$, and a stochastic forcing. For the IND experiment, $g=h=0$ meaning that $B$ and $N$ evolved independently. In terms of $F_{\text {CAM }}$, the IND case is equivalent to forcing the atmosphere 
Table 1 For each experiment: a description, the settings of the scaling parameters $g$ and $h$ (see Eq. 2), and the notation used to refer to the experiment

\begin{tabular}{llll}
\hline Description & $g$ & $h$ & Notation \\
\hline Independent $B$ and $N$ & 0 & 0 & IND \\
Autoregressive $B$ & 0 & 1 & AR \\
Vector autoregressive $B$ & 1 & 1 & CTL \\
Enhanced vector autoregressive $B$ & 2 & 1 & VAR2 \\
\hline
\end{tabular}

with an anomaly-free sea ice climatology. For the AR experiment, $B$ evolved as an autoregressive process, meaning it was sensitive to the past states of itself and a stochastic forcing. For the VAR2 experiment, $B$ evolved as in CTL but with a doubled sensitivity to $N$ (i.e., $g=2$ ). In the physical system, a change in the responsiveness of $B$ to $N$ could be related to, for example, changes in the thickness or extent of the sea ice.

For $F_{\mathrm{CAM}}$, we developed a 150 -member ensemble for each of CTL, IND, AR, and VAR2. Each member covered the 21 weeks beginning on December 4, with initial conditions taken from a long unforced run. We define the response as the total variance of the experiment ensemble divided by the total variance of the CTL ensemble. For $F_{\mathrm{VAR}}$, we define the response as the variance in the experiment case divided by the variance of the CTL case, where each variance comes from the solution to the linear system (6-7). We denote the response by a vertical bar followed by a subscript denoting the experiment. For example, the response of $\gamma_{N N}$ in the AR experiment is $\gamma_{N N \mid A R}$.

\section{Results}

We have two results sections. In the first (4.1), we present observations of $N$ and $B$, fit the VAR to these observations, and verify that the $F_{\mathrm{VAR}}$ and $F_{\mathrm{CAM}}$ models reasonably capture the observed behavior of $B$ and $N$. In Sect. 4.2, we present results from experimentation with $F_{\mathrm{VAR}}$ and $F_{\mathrm{CAM}}$.

\subsection{Observations}

For observations, the lagged correlations of $N$ and $B$ are shown in Fig. 2, and we will interpret them in the context of a hypothetical, anomalously high value of $N$. Concurrent with this high value of $N$ (at lag 0 ), $B$ tends to be anomalously low, indicating a reduction in sea ice over the Barents Sea that is physically consistent with the patterns of temperature advection and sea ice velocity associated with the positive NAO. This tendency for anomalously low $B$ is visible over lags of several weeks forward from the $N$ anomaly. One to six weeks after $B$ is anomalously low, $N$ tends to decrease (positive correlations toward left side of Fig. 2a). This is evidence of the negative feedback between $N$ and $B$ discussed in Sect. 1. Comparison of Fig. $2 \mathrm{~b}$ and c shows that $B$ is more autocorrelated than $N$ and subject to less short-term fluctuations or "noise."

Fitting system (2) to observations, we find the appropriate model order to be $p=4$ (method in Sect. 3.1) as in SMS. Parameter values for $\boldsymbol{\phi}$ matrix are as given in Table 2. By testing the significance of these parameters using Eq. 4, we conclude at the $95 \%$ confidence level that there is Granger feedback (Granger 1969) between $N$ and $B$. SMS provide a closely related result and more discussion of Granger feedback detection in this application. The blue and red curves in Fig. 2 show, respectively, lagged correlations for output from $F_{\mathrm{VAR}}$ and $F_{\mathrm{CAM}}$. Both models capture the temporal covariation of $N$ and $B$ reasonably well.

\subsection{Model responses}

We first use $F_{\mathrm{VAR}}$ to show how $\gamma_{N N}$ and $\gamma_{B B}$ respond to the $g$ and $h$ feedback scaling parameter settings in the IND, $\mathrm{AR}$, and VAR2 experiments. As noted in Sect. 3.1, we can
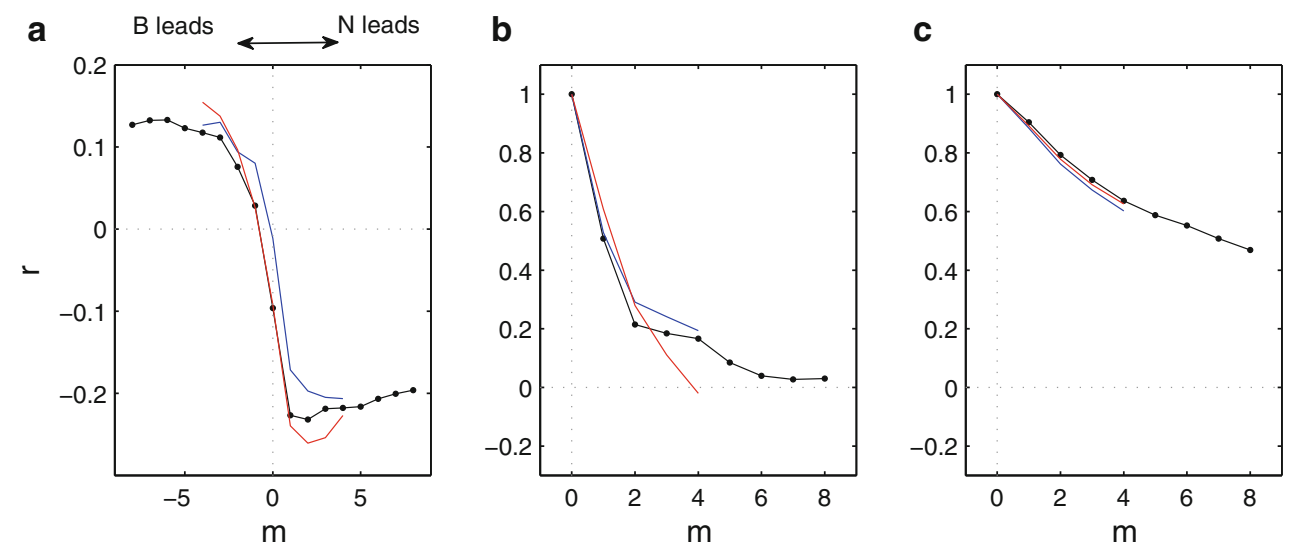

Fig. 2 Lagged Pearson correlation coefficients $r$ for (a) $B$ and $N$, (b) $N$ with itself, and (c) $B$ with itself. In each panel, the black curve with filled circles represent observations, the blue curve represents $F_{\mathrm{VAR}}$ model output, and the red curve represents $F_{\mathrm{CAM}}$ model output 
Table 2 Values of the parameters in the $\phi$ matrices of system (3) estimated from observations with $g=h=1$

\begin{tabular}{rrrrr}
\hline & $i=1$ & \multicolumn{1}{c}{$i=2$} & \multicolumn{1}{c}{$i=3$} & \multicolumn{1}{c}{$i=4$} \\
\hline$\alpha_{i}$ & 0.95 & -0.15 & 0.05 & 0.05 \\
$\beta_{i}$ & -0.16 & 0.06 & -0.03 & 0.01 \\
$\eta_{i}$ & 0.17 & -0.21 & 0.31 & -0.17 \\
$\phi_{i}$ & 0.55 & -0.12 & 0.12 & 0.07 \\
\hline
\end{tabular}

For the $\Upsilon$ matrix, the variance of $\varepsilon_{B}$ is 0.40 and the variance of $\varepsilon_{N}$ is 0.84

Table 3 Covariances for the system (3) solved for using observational estimates of the $\phi$ and $\Upsilon$ matrices in Table 2

\begin{tabular}{lr}
\hline$\gamma_{N N}$ & 1.02 \\
$\gamma_{B B}$ & 0.84 \\
$\gamma_{B N}(3)$ & -0.18 \\
\hline
\end{tabular}

directly obtain these responses by solving the linear system (6-7) with different values for $g$ and $h$. For the CTL case, we solved Eqs. 6-7 with $g=h=1$, and Table 3 provides some select values from this solution: $\gamma_{N N}, \gamma_{B B}$, and $\gamma_{B N}(3)$. Figure 3 shows that synthetic data generated from $F_{\text {VAR }}$ converge toward these numerical solutions as $t$ becomes large.

To provide a context for the three experiment results, we calculated $\gamma_{N N}$ and $\gamma_{B B}$ responses for a large set of values of $g$ and $h$ ranging from -1 to 2.5 (Fig. 4a, b, respectively). Figure $4 \mathrm{c}$ is a schematic indicating key locations or regions in response plane of $g$ and $h$. The open circles mark the locations of the CTL case and the three experiments IND, $\mathrm{AR}$, and VAR2. The responses in Fig. $4 \mathrm{a}$ and $\mathrm{b}$ are both 1.0 for the CTL case by definition.

In the AR experiment, feedback is turned off by setting $g=0$ meaning that $B$ is independent of previous values of $N$, but $N$ still depends on past values of $B$. The response is a $5 \%$ increase in $\gamma_{N N}$ (i.e., $\gamma_{N N \mid A R}=1.05$, Fig. $4 \mathrm{a}$ ). To provide the details underlying $\gamma_{N N \mid A R}$, we write the equation for $\gamma_{N N}$ from (7)

$\gamma_{N N}=\sum_{i=1}^{4}\left[h \eta_{i} \gamma_{N B}(i)+\phi_{i} \gamma_{N N}(i)\right]+\sigma_{N}^{2}$.

The $\gamma_{N B}(i)$ terms all become positive in AR meaning that anomalies of $B$ tend to be followed by like-signed anomalies of $N$, and the $\gamma_{N N}(i)$ terms increase, meaning that the autocorrelation of $N$ increases. Both changes contribute toward higher $\gamma_{N N}$. In the same experiment, the variance of $B$ decreases by $6 \%\left(\gamma_{B B \mid A R}=0.94\right.$, Fig. $\left.4 \mathrm{~b}\right)$. In the equation for $\gamma_{B B}$ from (7)

$\gamma_{B B}=\sum_{i=1}^{4}\left[\alpha_{i} \gamma_{B B}(i)+g \beta_{i} \gamma_{B N}(i)\right]+\sigma_{B}^{2}$,

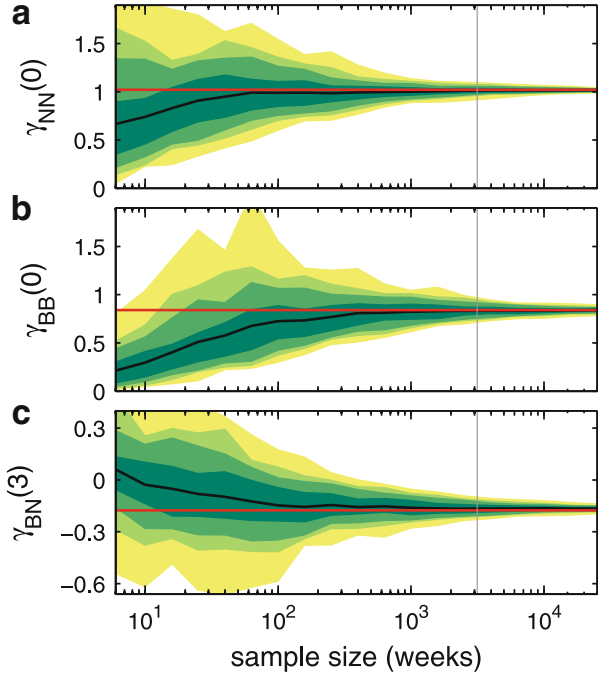

Fig. 3 Output from $F_{\mathrm{VAR}}$ converging to solutions of linear system (6-7) as $t$ becomes large. Variables are (a) the variance of $N$, (b) the variance of $B$, and (c) the covariance where $N$ leads $B$ by 3 weeks. The red line shows the solutions and the black curve shows the mean of 100 simulations. The distribution of results at each week $t$ is shaded in 10-percentile increments (i.e., yellow is 10-90th percentile, light green is 20-70th percentile, etc.). The vertical lines at $t=3,150$ show the number of weeks in each $F_{\mathrm{CAM}}$ ensemble

setting $g=0$ reduces the variance of $B$ because the $\gamma_{B N}$ terms contribute positively to $\gamma_{B B}$ in CTL.

In the VAR2 experiment, we increase the strength of the negative feedback by setting $g=2$. The variance, $\gamma_{N N}$, decreases by approximately $2 \%\left(\gamma_{N N \mid V A R 2}=0.98\right.$, Fig. $\left.4 \mathrm{a}\right)$ and $\gamma_{B B}$ increases by approximately $42 \%\left(\gamma_{B B \mid V A R 2}=1.42\right.$, Fig. 4b). In the IND experiment, the settings $g=h=0$ isolate $N$ and $B$ from each other, and the variance of each decreases $\left(\gamma_{N N \mid I N D}=0.99\right.$ in Fig. $4 \mathrm{a}, \gamma_{B B \mid I N D}=0.93$ in Fig. 4b). The variance responses for the IND case are not large, and arise from the significant but small feedback captured by fitting the $\boldsymbol{\phi}_{i}$ matrix in (3) to observational data. At the end of this section, we show that the variance responses in the $F_{\text {CAM }}$ results are larger.

Commenting more generally on the surfaces in Fig. 4, the responses become rapidly large in portions of the response plane of $g$ and $h$ that are shaded in Fig. 4c. In these shaded regions, the sign of either $g$ or $h$ becomes negative, rendering the feedback positive and generating very large variances of $N$ and $B$. The response surfaces are symmetrical about the origin with asymptotes (dashed lines) along which the contoured response is isolated from the other system variable, as in the IND case. Using the $\gamma_{N N}$ response as an example (Fig. $4 \mathrm{a}$ ), there is an asymptote at $h=0$ because this scaling desensitizes $N$ to $B$, so a change in $g$, which is a scaling factor in the $B$ equation, has no effect on $N$. 
Fig. 4 For $F_{\mathrm{VAR}}$ output, the response of (a) the variance of $N$ (denoted $\gamma_{N N}$ ) and (b) the variance of $B$ (denoted $\gamma_{B B}$ ) to changes in the feedback scaling parameters $g$ and $h$, and (c) a schematic indicating key areas in the response plane of $g$ and $h$. Further details in the text
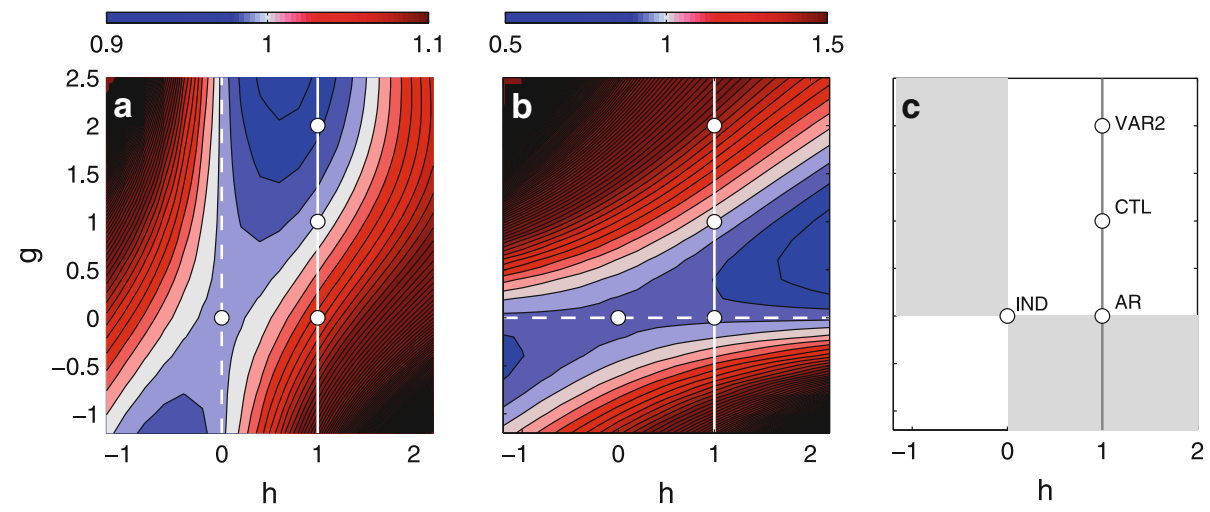

We now turn to the $F_{\mathrm{CAM}}$ results (red circles, Fig. 5). For the purposes of comparison, Fig. 5 shows portions of the $F_{\mathrm{VAR}}$ results as curves. These $F_{\mathrm{VAR}}$ response curves are taken from the surfaces in Fig. 4 running down the plots from VAR2 to CTL to AR (curve in Fig. 5a taken from Fig. 4a; curve in Fig. 5b taken from Fig. 4b). Considering the AR, CTL, and VAR2 results, the $F_{\mathrm{CAM}}$ results agreed qualitatively with the $F_{\mathrm{VAR}}$ results: turning feedback off in AR increases $\gamma_{N N}$ and decreases $\gamma_{B B}$, whereas doubling the feedback sensitivity in VAR2 produces the opposite responses. $F_{\mathrm{VAR}}$ models an approximately linear response of $\gamma_{N N}$ to $g$ (curve in Fig. 5a), and this curve is within one standard error of the $F_{\mathrm{CAM}}$ results. $F_{\mathrm{VAR}}$ models a strongly nonlinear response of $\gamma_{B B}$ to $g$ (curve in Fig. 5b), but the $F_{\mathrm{CAM}}$ results lack the curvature of the $F_{\mathrm{VAR}}$ model, suggesting an approximately linear response of $\gamma_{B B}$ to $g$. Linear regressions of the variance responses in $F_{\mathrm{CAM}}$ are shown as dashed lines in Fig. 5, and we conclude that the variance of $N$ and $B$ in $F_{\mathrm{CAM}}$ depend approximately linearly on the sensitivity of $B$ to $N$ for the range $0 \leq g \leq 2$.

For the IND case, the $\gamma_{B B}$ response is the same as in the AR case (not shown) because $B$ lacks sensitivity to $N$ in both experiments (i.e., $g=0$, Table 1). For $\gamma_{N N}$ in the IND case, $h=0$ and the $F_{\text {CAM }}$ result matches in sign, but is stronger than the $F_{\mathrm{VAR}}$ result (Fig. 5a). Specifically, $F_{\mathrm{VAR}}$ predicts a small $\gamma_{N N}$ response when isolating $N$ from $B$, but $F_{\text {CAM }}$ produces the strongest response in $\gamma_{N N}$ for the cases we examined, amounting to a $6 \%$ decrease. $F_{\mathrm{VAR}}$ and $F_{\mathrm{CAM}}$ are thus in agreement about responses with respect to the sensitivity of $B$ to $N$ (i.e., $g$ ), but agree less well about responses with respect to the sensitivity of $N$ to $B$ (i.e., $h$ ). This is not entirely unexpected since $F_{\text {CAM }}$ generates $N$ using nonlinear equations of motion and parameterized model physics.

\section{Summary and discussion}

We quantified the effects of negative feedback between the North Atlantic Oscillation index (denoted $N$ ) and an index
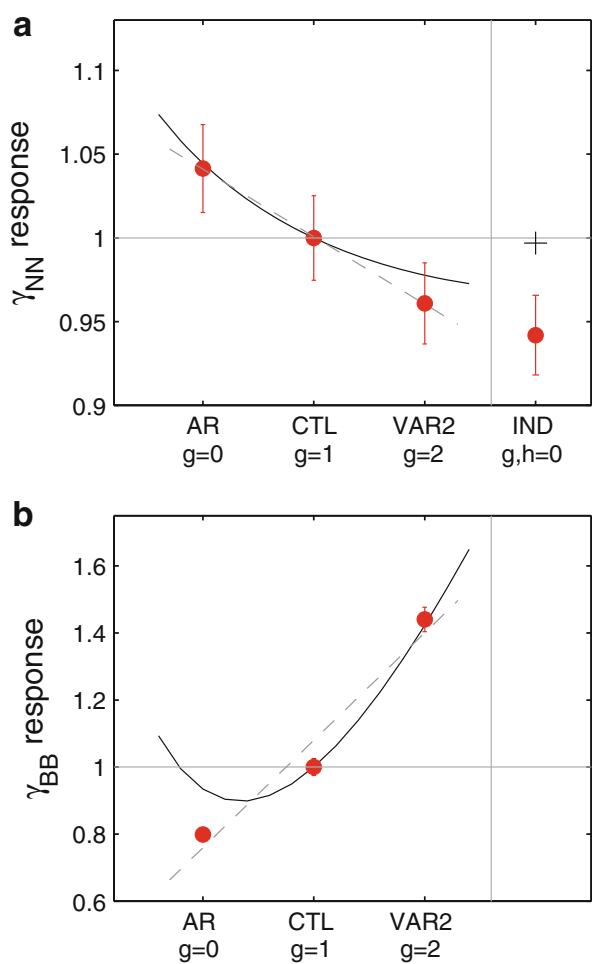

Fig. 5 a For the variance of $N$ (denoted $\gamma_{N N}$ ), the curve and cross show the responses given by $F_{\mathrm{VAR}}$ for the four cases indicated on the abscissa. The red circles show the responses produced by $F_{\text {CAM }}$ with error bars showing plus or minus one standard error. The dashed line is a least squares linear fit to the AR, CTL and VAR2 results from $F_{\text {CAM }}$ as a function of $g$. b Same as a, but for the variance of $B$ (denoted $\gamma_{B B}$ ), and the IND case is not show because it is equivalent to the AR case

of sea ice concentration anomalies in and around the $\mathrm{Ba}$ rents Sea (denoted $B$ ). Statistically testable definitions of causality and feedback were used to conclude that, in observations, positive $N$ tends to produce negative $B$, which in turn forces negative $N$. We then investigated this feedback by modifying it in two modeling frameworks: a statistical vector autoregressive model $\left(F_{\mathrm{VAR}}\right)$ and an atmospheric global climate model $\left(F_{\mathrm{CAM}}\right)$ customized so that sea ice anomalies on the lower boundary were 
stochastic with adjustable sensitivity to the model's evolving $N$. We focused on how the variance of $N$ and the variance of $B$ respond to changes in the system feedback.

We defined a control case (CTL) in which $B$ evolved as a vector autoregressive process sensitive to the past states of itself, the past states of $N$, and a stochastic forcing. For the IND experiment, $B$ and $N$ evolved independently. For the AR experiment, $B$ evolved as an autoregressive process, meaning it was sensitive to the past states of itself and a stochastic forcing. For the VAR2 experiment, $B$ evolved as in CTL but with a doubled sensitivity to $N$. In the physical system, a change in the responsiveness of $B$ to $N$ could be related to interannually varying properties of the sea ice. For example, a thinner sea ice pack could be more responsive to thermodynamic forcing by NAO-driven atmospheric temperature advection. Also, the position of the sea ice edge relative to the centers of action of the NAO could govern how sensitive $B$ is to wind-driven sea ice advection.

In the following conclusions, we take "feedback strength" to mean the value of the feedback scaling parameter $g$, which is the sensitivity of $B$ to $N$. The variance of $N\left(\gamma_{N N}\right)$ tends to decrease as feedback strength increases in $F_{\mathrm{CAM}}$ and $F_{\mathrm{VAR}}$, and this sensitivity depends approximately linearly on $g$. The variance of $B\left(\gamma_{B B}\right)$ tends to increase as feedback strength increases in $F_{\mathrm{CAM}}$ and $F_{\mathrm{VAR}}$, and this sensitivity is approximately linear in $F_{\mathrm{CAM}}$, exhibiting more curvature in $F_{\mathrm{VAR}}$. In $F_{\mathrm{CAM}}$, the IND case produced the strongest response in $\gamma_{N N}$, amounting to a $6 \%$ decrease in variance. This is different from the $F_{\mathrm{VAR}}$ prediction that $\gamma_{N N}$ would be very similar in the IND and CTL cases. This difference indicates that $F_{\mathrm{VAR}}$ and $F_{\mathrm{CAM}}$ produce reasonably similar responses when feedback is scaled by changing the sensitivity of $B$ to $N$ (i.e., $g$ ), but produce less similar responses when feedback is scaled by changing the sensitivity of $N$ to $B$.

Based on the $F_{\text {CAM }}$ results, the variance of $N$ increased progressively from IND to VAR2 to CTL to AR. In other words, a zero-anomaly sea ice climatology (IND case) produces minimal $N$ variance whereas, given that the sea ice field has anomalies (VAR2, CTL, or AR cases), the variance of $N$ goes down as these anomalies become more sensitive to $N$. This negative-slope, approximately linear response of $\gamma_{N N}$ to the feedback strength is consistent with the fundamental behavior of negative feedback, and its quantification has implications for the simulation of internal variability in atmospheric global climate models forced by sea ice, and for the predictability of $N$ under projected changes in winter sea ice extent.

Acknowledgments This research was supported by NSF grant ATM-0612779 and NOAA grants NA06OAR4310149 and NA09OAR4310132. The manuscript was improved by discussions with Hal Stern, as well as comments from two anonymous reviewers.
Open Access This article is distributed under the terms of the Creative Commons Attribution Noncommercial License which permits any noncommercial use, distribution, and reproduction in any medium, provided the original author(s) and source are credited.

\section{References}

Alexander MA, Bhatt US, Walsh JE, Timlin MS, Miller JS, Scott JD (2004) The atmospheric response to realistic Arctic sea ice anomalies in an AGCM during winter. J Clim 17:890-905

Brockwell PJ, Davis RA (1996) Introduction to time series and forecasting. Springer, New York

Cavalieri D, Parkinson C, Gloersen P, Zwally HJ (2008) Sea ice concentrations from Nimbus-7 SMMR and DMSP SSM/I passive microwave data, 1978-2008. National Snow and Ice Data Center, Boulder, Colorado. Digital media

Comiso JC (2006) Abrupt decline in Arctic winter sea ice cover. Geophys Res Lett 33. doi:10.1029/2006GL027341

Delworth TL, Manabe S, Stouffer RJ (1997) Multidecadal climate variability in the Greenland Sea and surrounding regions: a coupled model study. Geophys Res Lett 24:257-260. doi: 10.1029/96GL03927

Deser C (2000) On the teleconnectivity of the "Arctic Oscillation". Geophys Res Lett 27:779-782

Deser C, Teng H (2008) Evolution of Arctic sea ice concentration trends and the role of atmospheric circulation forcing, 19792007. Geophys Res Lett 35. doi:10.1029/2007GL032023

Deser C, Walsh JE, Timlin MS (2000) Arctic sea ice variability in the context of recent atmospheric circulation trends. J Clim 13:617-633

Deser C, Magnusdottir G, Saravanan R, Phillips AS (2004) The effects of North Atlantic SST and sea-ice anomalies on the winter circulation in CCM3. Part II: Direct and indirect components of the response. J Clim 17:877-889

Deser C, Tomas RA, Peng S (2007) The transient atmospheric circulation response to North Atlantic SST and sea ice anomalies. J Clim 20:4751-4767

Fauria MM, Grinsted A, Helama S, Moore J, Timonen M, Martma T, Isaksson E, Eronen E (2009) Unprecedented low twentieth century winter sea ice extent in the Western Nordic Seas since A.D. 1200. Clim Dyn. doi:10.1007/s00382-009-0610-z

Francis JA, Hunter E (2004) Drivers of declining sea ice extent in the Arctic winter: a tale of two seas. Geophys Res Lett 61:145-160

Granger CWJ (1969) Investigating causal relationships by econometric models and cross-spectral methods. Econometrica 37:473-485

$\mathrm{Hu}$ A, Rooth C, Bleck R, Deser C (2002) NAO influence on sea ice extent in the Eurasian coastal region. Geophys Res Lett 29. doi: 10.1029/2001GL014293

Hurrell JW (1995) Decadal trends in the North Atlantic Oscillation: regional temperatures and precipitation. Science 269:676-679

Johannessen OM, Bengtsson L, Miles MW, Kuzmina SI, Semenov VA, Alekseev GV, Nagurnyi AP, Zakharov VF, Boblyev LP, Petterson LH, Hasselmann K, Cattle HP (2004) Arctic climate change: observed and modelled temperature and sea-ice variability. Tellus 56:328-341

Jungcalus JH, Haak H, Latif M, Mikolajewicz U (2005) Arctic-North Atlantic interactions and multidecadal variability of the meridional overturning circulation. J Clim 18:4013-4031

Kimura N, Wakatsucchi M (2001) Mechanism for the variation of sea ice extent in the Northern Hemisphere. J Geophys Res 106:31,319-31,331

Koenigk T, Mikolajewicz U, Jungclaus JH, Kroll A (2009) Sea ice in the Barents Sea: seasonal to interannual variability and climate feedbacks in a global coupled model. Clim Dyn 32:1119-1138 
Kvamsto NG, Skeie P, Stephenson DB (2004) Impact of Labrador sea-ice extent on the North Atlantic Oscillation. Int J Climatol 24:603-612

Kwok R, Maslowski W, Laxon SW (2005) On large outflows of Arctic sea ice into the Barents Sea. Geophys Res Lett 33. doi: 10.1029/2005GL024485

Liu J, Curry JA (2004) Recent Arctic sea ice variability: connections to the Arctic Oscillation and the ENSO. Geophys Res Lett 31. doi:10.1029/2004GL019858

Magnusdottir G, Deser C, Saravanan R (2004) The effects of North Atlantic SST and sea ice anomalies on the winter circulation in CCM3. Part I: main features and storm track characteristics of the response. J Clim 17:857-876

Maslanik J, Drobot S, Fowler C, Emery W, Barry R (2007) On the Arctic climate paradox and the continuing role of atmospheric circulation in affecting sea ice conditions. Geophys Res Lett 34. doi:10.1029/2006GL028269

Rigor IG, Wallace JM, Colony RL (2002) Response of sea ice to the Arctic Oscillation. J Clim 15:2648-2663

Rothrock DA, Zhang J (2005) Arctic ocean sea ice volume: What explains its recent depletion? J Geophys Res 110. doi: 10.1029/2004JC002282

Serreze MC, Holland MM, Stroeve J (2007) Perspectives on the arctic's shrinking sea-ice cover. Science 315:1533-1536
Sims CA (1980) Macroeconomics and reality. Econometrica 48:1-48

Strong C, Magnusdottir G (2010a) Modeled winter sea ice variability and the North Atlantic Oscillation: a multi-century perspective. Clim Dyn 34(4):515-525. doi:10.1007/s00382-009-0550-7

Strong C, Magnusdottir G (2010b) The role of Rossby wave breaking in shaping the equilibrium atmospheric circulation response to North Atlantic boundary forcing. J Clim 23:1269-1276

Strong C, Magnusdottir G, Stern H (2009) Observed feedback between winter sea ice and the North Atlantic Oscillation. J Clim 22:6021-6032

Ukita J, Honda M, Nakamura H, Tachibana Y, Cavlieri DJ, Parkinson CL, Koide H, Yamamoto K (2007) Northern Hemisphere sea ice variability: Lag structure and its implications. Tellus, Ser A 59:261-272. doi:10.111/j.1600-0870.2006.0223.x

Venegas SA, Mysak LA (2000) Is there a dominant timescale of natural climate variability in the Arctic?. J Clim 13:3412-3434

Wang J, Wu B, Tang C, Walsh JE, Ikeda M (2000) Seesaw structure of subsurface temperature anomalies between the Barents Sea and the Labrador Sea. Geophys Res Lett 31. doi:10.1029/2004 GL019981

Yamamoto K, Tachibana Y, Honda M, Ukita J (2006) Possible feedback of winter sea ice in the Greenland and Barents Seas on the local atmosphere. Geophys Res Lett 33. doi:10.1029/2006 GL026286 